\title{
Smectic Layered Polymer Networks Based on Novel Side Chain Liquid Crystalline Polymers Having Thermally Reversible Urea Bonds
}

\author{
Sang Hyuk Seo, Young-Woon Kim, and Ji Young Chang* \\ Hyperstructured Organic Materials Research Center, School of Materials Science and \\ Engineering, Seoul National University, Seoul 151-744, Korea.
}

\section{Supporting Information}

Synthesis of 1 . Compound 1 was synthesized according to the published method (Bruice, T. C.; Herz, J. L. J. Am. Chem. Soc. 1964, 86, 4109) and used for the next reaction without further purification. ${ }^{1} \mathrm{H}$ NMR $\left(300 \mathrm{MHz}, \mathrm{DMSO}-d_{6}\right) \delta(\mathrm{ppm}) 8.85$, 8.23 (s, $2 \mathrm{H}$; imidazole ring protons).

Synthesis of 2a-2b. 4, 4'-Biphenol (2 g, $10 \mathrm{mmol}$ ) was dissolved in a solution of 1.2 $\mathrm{g}$ of potassium hydroxide, $5 \mathrm{ml}$ of water, $15 \mathrm{ml}$ of ethanol, and $0.16 \mathrm{~g}$ of potassium iodide. After refluxing for $1 \mathrm{~h}, n$-bromoalkane $(\mathrm{n}=6$ or 8$)(15 \mathrm{mmol})$ was added dropwise. The solution was refluxed for $24 \mathrm{~h}$. After filtration and evaporation, the product was isolated by column chromatography on silica gel (THF : $n$-hexane $=1: 5$ ).

Yield: $65 \%$ for $\mathbf{2 a}$ and $58 \%$ for $\mathbf{2 b .} \mathbf{2 a :}{ }^{1} \mathrm{H}$ NMR (300 $\left.\mathrm{MHz}, \mathrm{CDCl}_{3}\right) \delta(\mathrm{ppm}) 6.88,6.93$, 7.43 (dd, 8H; ArH), 4.70 (s, 1H; OH), 3.99 (t, 2H; $\left.\mathrm{ArOCH}_{2}\right), 1.32-1.80\left(\mathrm{~m}, 8 \mathrm{H} ; \mathrm{CH}_{2}\right)$, $0.91\left(\mathrm{t}, 3 \mathrm{H} ; \mathrm{CH}_{3}\right) . \mathbf{2 b}:{ }^{1} \mathrm{H}$ NMR $\left(300 \mathrm{MHz}, \mathrm{CDCl}_{3}\right) \delta(\mathrm{ppm}) 6.88,6.94,7.44(\mathrm{dd}, 8 \mathrm{H}$; ArH), $4.74(\mathrm{~s}, 1 \mathrm{H} ; \mathrm{OH}), 3.98\left(\mathrm{t}, 2 \mathrm{H} ; \mathrm{ArOCH}_{2}\right), 1.31-1.84\left(\mathrm{~m}, 12 \mathrm{H} ; \mathrm{CH}_{2}\right), 0.88(\mathrm{t}, 3 \mathrm{H}$; $\mathrm{CH}_{3}$ ).

Synthesis of 3a-3b. To a solution of compound $\mathbf{2 a}$ or $\mathbf{2 b}(7.4 \mathrm{mmol})$ in dry DMF (50 
$\mathrm{ml}$ ) was added sodium hydride $(0.224 \mathrm{~g}, 8.8 \mathrm{mmol})$. After stirring for $2 \mathrm{~h}$ at room temperature, compound $\mathbf{1}$ (1.24 g, $7.4 \mathrm{mmol})$ was added. The solution was stirred for 30 $\mathrm{h}$ at $90{ }^{\circ} \mathrm{C}$. After filtration and evaporation, the product was isolated by column chromatography on silica gel (THF : $n$-hexane $=5: 1$ ) and further purified by recrystallization from THF/n-hexane. Yield: $45 \%$ for $\mathbf{3 a}$ and $41 \%$ for $\mathbf{3 b}$. 3a: ${ }^{1} \mathrm{H}$ NMR $\left(300 \mathrm{MHz}, \mathrm{DMSO}-d_{6}\right) \delta(\mathrm{ppm}) 12.86(\mathrm{~s}, 1 \mathrm{H} ; \mathrm{NH}), 7.91,8.10(\mathrm{~s}, 2 \mathrm{H}$; imidazole ring protons), 7.01, 7.24, $7.64(\mathrm{dd}, 8 \mathrm{H} ; \mathrm{ArH}), 4.00\left(\mathrm{t}, 2 \mathrm{H} ; \mathrm{ArOCH}_{2}\right), 1.29-1.75\left(\mathrm{~m}, 8 \mathrm{H} ; \mathrm{CH}_{2}\right)$, $0.86\left(\mathrm{t}, 3 \mathrm{H} ; \mathrm{CH}_{3}\right) ;{ }^{13} \mathrm{C}$ NMR (300 MHz, DMSO- $\left.d_{6}\right) \delta(\mathrm{ppm}) 158.6,157.6,156.6,149.7$, $137.5,132.5,127.7,127.0,122.0,115.8,115.3,115.1,68.0,30.9,28.7,25.1,21.8,13.6$; IR (KBr): $v=3424,3038,2931,1733,1609,1210,798$; Anal. Calcd. for $\mathrm{C}_{22} \mathrm{H}_{24} \mathrm{~N}_{2} \mathrm{O}_{3}$ : C, 72.50; H, 6.64; N, 7.69. Found: C, 72.46; H, 6.72; N, 7.69. 3b: ${ }^{1} \mathrm{H}$ NMR (300 MHz, DMSO- $\left.d_{6}\right) \delta(\mathrm{ppm}) 12.82(\mathrm{~s}, 1 \mathrm{H} ; \mathrm{NH}), 7.90,8.09$ (s, 2H; imidazole ring protons), 7.02, 7.25, 7.65 (dd, 8H; ArH), 4.01 (t, 2H; $\left.\mathrm{ArOCH}_{2}\right), 1.19-1.75\left(\mathrm{~m}, 12 \mathrm{H} ; \mathrm{CH}_{2}\right), 0.85$ (t, 3H; $\left.\mathrm{CH}_{3}\right) ;{ }^{13} \mathrm{C}$ NMR (300 MHz, DMSO- $\left.d_{6}\right) \delta(\mathrm{ppm}) 158.2,157.4,156.3,149.2,137.2,131.5$, $127.5,126.9,122.0,115.4,114.8,114.6,67.4,30.5,28.2,28.0,27.9,24.9,21.3,13.1$; IR $(\mathrm{KBr}): v=3423,3039,2926,1735,1609,1208,798$; Anal. Calcd. for $\mathrm{C}_{24} \mathrm{H}_{28} \mathrm{~N}_{2} \mathrm{O}_{3}$ : C, 73.44; H, 7.19; N, 7.14. Found: C, 73.10; H, 7.19; N, 7.10.

Synthesis of 4a-4b. Compound $\mathbf{3 a}$ or $\mathbf{3 b}(1.37 \mathrm{mmol})$ and 2-isocyanatoethyl methacrylate (IEM) $(0.4 \mathrm{ml}, 2.7 \mathrm{mmol})$ were dissolved in dry THF $(20 \mathrm{ml})$ and then the solution was stirred for $7 \mathrm{~h}$ at $40{ }^{\circ} \mathrm{C}$. The product was isolated by precipitation in $n$ hexane. Yield: $94 \%$ for $\mathbf{4 a}$ and $91 \%$ for $\mathbf{4 b .} \mathbf{4 a}:{ }^{1} \mathrm{H}$ NMR $\left(300 \mathrm{MHz}, \mathrm{CDCl}_{3}\right) \delta(\mathrm{ppm})$ 8.86 (t, 1H; NHCON), 8.37, 8.58 (s, 2H; imidazole ring protons), 7.01, 7.26, 7.61 (dd, $8 \mathrm{H} ; \mathrm{ArH}), 6.06,5.67$ (s, 2H; vinyl protons), $4.02\left(\mathrm{t}, 2 \mathrm{H} ; \mathrm{ArCH}_{2} \mathrm{O}\right), 4.28,3.58(\mathrm{t}, 4 \mathrm{H}$; 
$\mathrm{COOCH}_{2} \mathrm{CH}_{2}$ ), 1.88 (s, 3H; vinyl- $\left.\mathrm{CH}_{3}\right), 1.31-1.73\left(\mathrm{~m}, 8 \mathrm{H} ; \mathrm{CH}_{2}\right), 0.88\left(\mathrm{t}, 3 \mathrm{H} ; \mathrm{CH}_{3}\right) ;{ }^{13} \mathrm{C}$ NMR (300 MHz, $\left.\mathrm{CDCl}_{3}\right) \delta(\mathrm{ppm}) 168.1,161.8,159.3,149.4,148.6,139.5,137.6,136.1$, $133.7,132.7,128.5,128.1,126.9,124.1,122.2,115.3,68.5,63.2,40.9,32.0,29.7,26.1$, 23.0, 18.6, 14.5; IR (KBr): $v=3232,3114,2954,1745,1721,1609,1170,800$; Anal. Calcd. for $\mathrm{C}_{29} \mathrm{H}_{33} \mathrm{~N}_{3} \mathrm{O}_{6}$ : C, 67.04; H, 6.40; N, 8.09. Found: C, 67.10; H, 6.45; N, 8.01. 4b: ${ }^{1} \mathrm{H}$ NMR (300 MHz, $\left.\mathrm{CDCl}_{3}\right) \delta(\mathrm{ppm}) 8.95(\mathrm{t}, 1 \mathrm{H}$; NHCON), 8.41, $8.61(\mathrm{~s}, 2 \mathrm{H}$; imidazole ring protons), 7.01, 7.25, 7.61 (dd, 8H; ArH), 6.07, 5.70 (s, 2H; vinyl protons), 4.01 (t, 2H; $\mathrm{ArCH}_{2} \mathrm{O}$ ), 4.27, 3.59 (t, 4H; $\mathrm{COOCH}_{2} \mathrm{CH}_{2}$ ), 1.88 (s, 3H; vinyl- $\mathrm{CH}_{3}$ ), 1.29$1.71\left(\mathrm{~m}, 12 \mathrm{H} ; \mathrm{CH}_{2}\right), 0.86\left(\mathrm{t}, 3 \mathrm{H} ; \mathrm{CH}_{3}\right) ;{ }^{13} \mathrm{C} \mathrm{NMR}\left(300 \mathrm{MHz}, \mathrm{CDCl}_{3}\right) \delta(\mathrm{ppm})$ 167.9, $161.4,158.9,149.1,148.1,139.1,137.1,135.7,133.6,132.4,128.1,127.8,126.7,123.4$, $121.8,114.9,68.2,62.9,40.8,31.9,29.4,29.3,29.2,26.1,22.7,18.2,14.1$; IR (KBr): v $=3232,3117,2931,1744,1723,1609,1170,801$; Anal. Calcd. for $\mathrm{C}_{31} \mathrm{H}_{37} \mathrm{~N}_{3} \mathrm{O}_{6}: \mathrm{C}$, 67.99; H, 6.81; N, 7.67. Found: C, 67.71; H, 6.77; N, 7.57.

Synthesis of 5a-5b. Compound $4 \mathbf{a}$ or $\mathbf{4 b}(0.37 \mathrm{mmol})$ and MMA $(0.078 \mathrm{ml}, 0.74$ mmol) were charged into a polymerization tube $(15 \mathrm{ml})$ and a solution of AIBN (18 mg, $0.01 \mathrm{mmol})$ in dry acetone $(4 \mathrm{ml})$ was added. After five freeze-thaw cycles, the tube was sealed under vacuum. The solution was stirred for $24 \mathrm{~h}$ at $60{ }^{\circ} \mathrm{C}$. The polymer was isolated by precipitation in $n$-hexane and purified by reprecipitation from the polymer solution in acetone into $n$-hexane twice. Yield: $83 \%$ for $\mathbf{5 a}$ and $88 \%$ for $\mathbf{5 b}$. About $65 \%$ of MMA units were found in both copolymers. 5a: ${ }^{1} \mathrm{H} \mathrm{NMR}\left(\mathrm{CDCl}_{3}, 300 \mathrm{MHz}\right) \delta$ (ppm) 8.53-7.53 (m, 3H; imidazole ring protons and $\mathrm{NHCON}), 7.50-6.96(\mathrm{~m}, 8 \mathrm{H}$; ArH), 4.30-3.30 (m, 6H; $\mathrm{ArCH}_{2} \mathrm{O}$ and $\mathrm{COOCH}_{2} \mathrm{CH}_{2}$ ), 0.8-2.0 (m, 13H; alkyl chain protons); IR $(\mathrm{KBr}): \mathrm{v}=3365,3140,2929,2275,1732,1608,1170,803.5 \mathbf{b}:{ }^{1} \mathrm{H}$ NMR $\left(\mathrm{CDCl}_{3}\right.$, 
$300 \mathrm{MHz}) \delta(\mathrm{ppm})$ 8.52-7.53 (m, 3H; imidazole ring protons and NHCON), 7.50-6.95 $(\mathrm{m}, 8 \mathrm{H} ; \mathrm{ArH}), 4.30-3.30\left(\mathrm{~m}, 6 \mathrm{H} ; \mathrm{ArCH}_{2} \mathrm{O}\right.$ and $\left.\mathrm{COOCH}_{2} \mathrm{CH}_{2}\right), 0.8-2.0(\mathrm{~m}, 17 \mathrm{H}$; alkyl chain protons); IR (KBr): $v=3368,3138,2929,2275,1732,1609,1170,802$.

Measurements. ${ }^{1} \mathrm{H}$ and ${ }^{13} \mathrm{C}$ nuclear magnetic resonance (NMR) spectra were measured by BRUKER Avance DPX-300 (300 MHz) spectrometer using $\mathrm{CDCl}_{3}$, DMSO- $d_{6}$ or DMF- $d_{7}$ as solvents. All chemical shifts were listed in ppm downfield from tetramethylsilane. Fourier transform infrared (FT-IR) measurements were recorded on a PERKIN ELMER Spectrum GX1 spectrometer with $\mathrm{KBr}$ pellets. The differential scanning calorimetry (DSC) measurements were performed by a TA modulated DSC 2920 at scanning rate of $5-10{ }^{\circ} \mathrm{C} / \mathrm{min}$. An optical microscopy study was performed by a Leica DM LP equipped with Mettler Toledo FP 82HT heating stage and Mettler Toledo FP 90 central process controller. Samples were prepared by placing solid powder samples onto a slide glass and cover glass. Elemental analyses were carried out on an EAGER 200 elemental analyzer at the National Center for Inter-University Research Facilities of Seoul National University, Seoul, Korea. Gel permeation chromatographic (GPC) analysis was conducted in tetrahydrofuran with a Viscotek Model 250 equipped with PL Gel Mix-B and 5- $\mu$ Mix-D columns at room temperature. Approximate calibration of the column was accomplished by means of narrow molecular weight polystyrene standards. Wide-angle and small-angle X-ray diffractogram were obtained using a Bruker Xps GADDS ( $\mathrm{Cu} \mathrm{K \alpha}$ radiation, $\lambda=1.54 \AA$ ) at the National Instrumentation Center for Environmental Management, Seoul National University, Suwon, Korea. TEM measurements were carried out on a CMC20 transmission electron microscope at $200 \mathrm{kV}$. Samples of networked material (5a) for TEM charactrization were first embedded in epoxy resin and cured at $60{ }^{\circ} \mathrm{C}$ overnight. Ultrathin sections 
(approximately $80 \mathrm{~nm}$ ) were microtomed at room temperature. Thereafter, $80 \mathrm{~nm}$ thin sections were picked up on 200 mesh copper grids. 


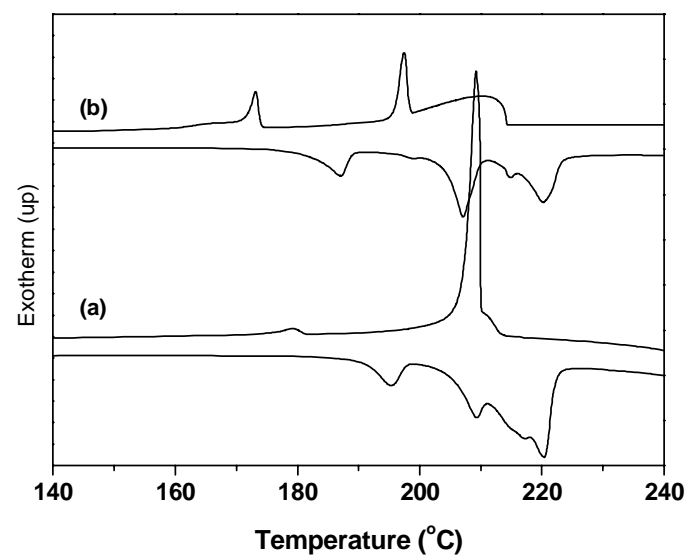

Figure 1. DSC thermograms of compounds 3a (a) and $\mathbf{3 b}$ (b) obtained with a heating rate of $10 \square / \mathrm{min}$ and a cooling rate of $10 \square / \mathrm{min}$ under nitrogen. For all compounds the second heating and cooling traces are given.

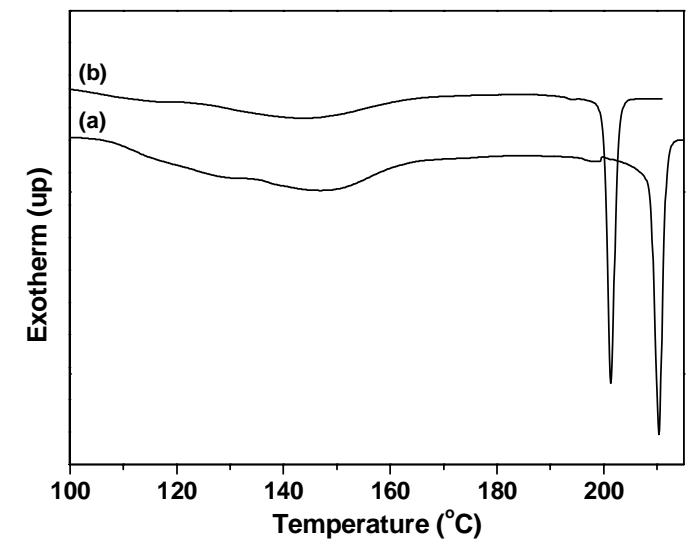

Figure 2. DSC thermograms of compounds $\mathbf{5 a}$ (a) and $\mathbf{5 b}$ (b) obtained with a heating rate of $10 \square / \mathrm{min}$ and a cooling rate of $10 \square / \mathrm{min}$ under nitrogen. For all compounds the first heating traces are given. 
(a)

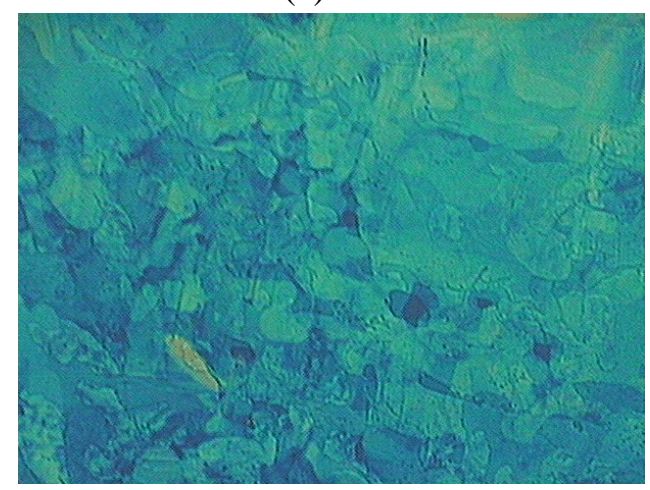

(c)

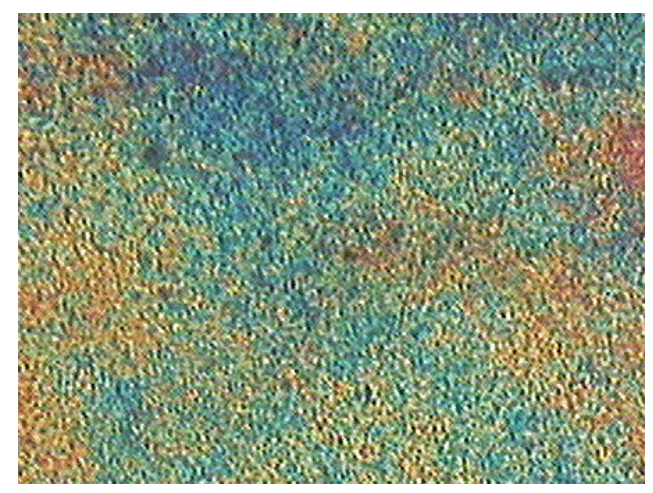

(b)

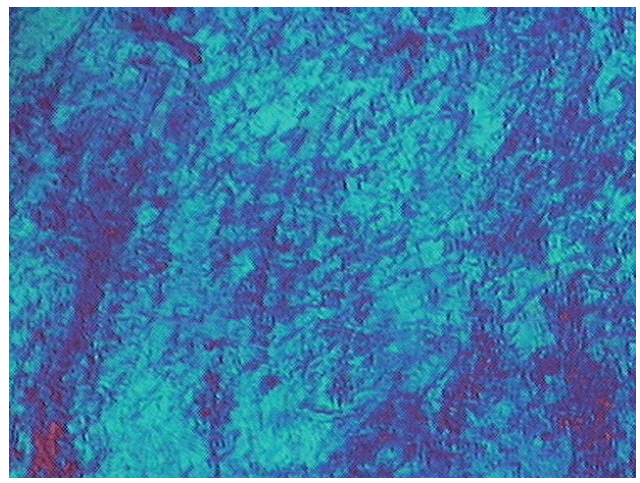

(d)

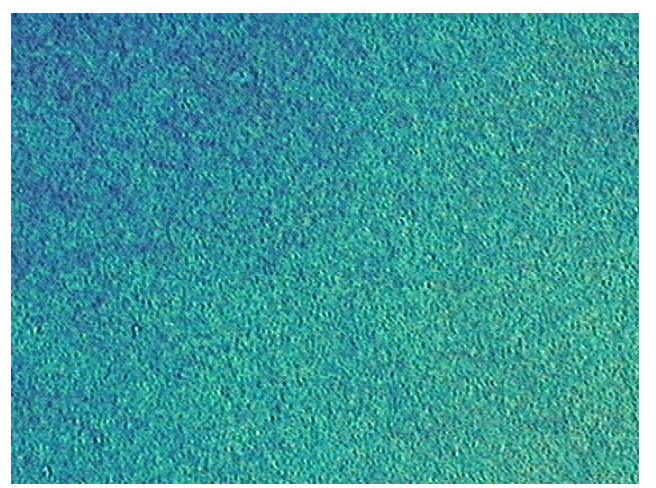

Figure 3. Polarized optical micrographs of compound 3a at $208 \square$ (a), 3b at $208 \square$ (b), 5a at $155 \square$ (c), and $\mathbf{5 b}$ at $153 \square$ (d) [Images (a) and (b) were obtained on second cooling, and (c) and (d) on first heating]. 


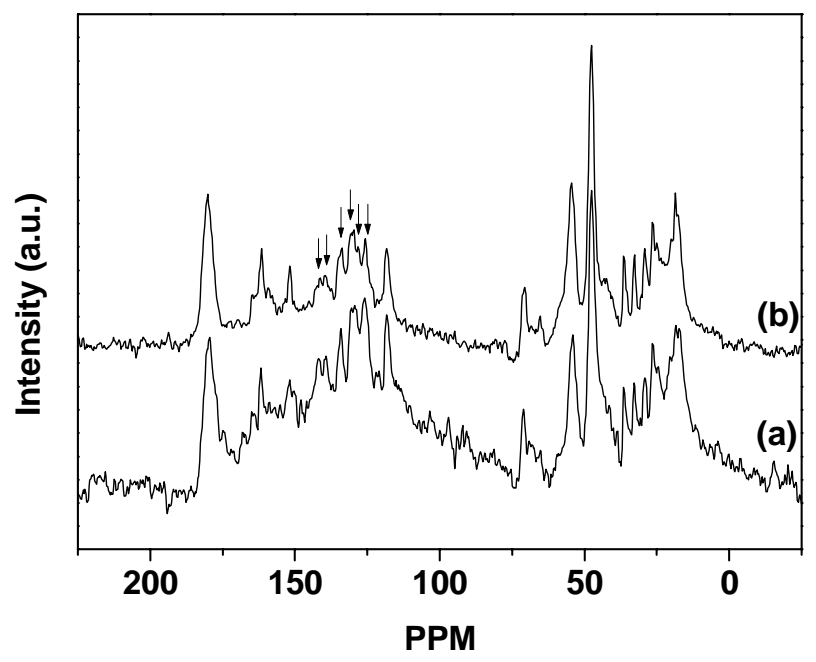

Figure 4. Solid-state ${ }^{13} \mathrm{C}$ NMR spectra of polymer 5a ; (a) annealed for $5 \mathrm{~h}$ at $155{ }^{\circ} \mathrm{C}$ and (b) washed in THF after annealing. 